\title{
IMPLICACIONES AMBIENTALES Y A LA SALUD DE LA NANOTECNOLOGÍA
}

\author{
Environmental and health implications of \\ nanotechnology
}

\author{
Gian Carlo Delgado-Ramos*
}

\section{Resumo}

La nanotecnología, o la capacidad de manipular la materia a escala nanométrica, promete perfilarse como el principal eje de la proxima revolución tecnológica centrada en lo que han calificado algunos como el inevitable avance de las tecnologías convergentes (biotecnología, nanotecnología, tecnologías de la información y ciencias cognitivas). Como tal, su impacto e implicaciones prometen ser mayores. En lo que refiere a las implicaciones ambientales y a la salud de la nanotecnología, el grado de incertidumbre y de riesgo apunta ser altamente inusitado. El presente texto discute algunos de los principales razonamientos que sustentan tal argumentación por medio de recuperar numerosos estudios de riesgo sobre las nanopartículas en el medio ambiente y sus interacciones. Asi mismo, se revisan los potenciales impactos del uso de nanoestructuras en lo que se ha calificado como "nanomedicina", los nano-cosméticos y la nanoagroindustria; todo, al tiempo que hace un llamado al amplio estudio y diálogo social sobre las implicaciones éticas, sociales y ambientales del desarrollo científico-tecnológico de nuestro tiempo.

Palabras clave: Nanotecnología. Medio ambiente. Salud.

* Economista egresado de la UNAM; maestría y doctorado en Ciencias Ambientales y Economía Ecológica de parte de la Universidad Autónoma de Barcelona, España. Email: giandelgado@gmail. com e giandelgado@hotmail.com 


\section{Abstract}

Nanotechnology, or the technical capacity of manipulating matter at the nanoscale, seems to be evolving as the main front of the next technological revolution. Centered on what some have called the inevitable advance of converging technologies (biotechnology, nanotechnology, information technologies and cognitive sciences), the impacts and implications of such revolution promise to be of major order. On what respects to environmental and health implications of nanotechnology, the degree of uncertainty and risk seems to be highly unexpected. The present text discusses some of the main related arguments recovering several risk studies on nanoparticles and their interactions with the environment. It also offers a review on the potential impacts of nanostructures' uses on what has been called "nanomedicine", the nano-cosmetics and the nano-agroindustry; all, while it makes a call to extend the studies, and social dialogue, on the ethical, societal and environmental implications of the scientific and technological development of our time.

Keywords: Nanotechnology. Environment. Health.

\section{INTRODUCCIÓN}

Las promesas que genera la nanotecnología son de tal magnitud que varios países y sus multinacionales, y el grueso de estados capitalistas centrales, están incrementando exponencialmente sus gastos en investigación al respecto. Se dice que entre los campos de operación de mayor potencial en el corto/mediano plazo figuran los que permiten: (1) la construcción de estructuras materiales novedosas, (2) aplicaciones en el procesamiento, almacenamiento y transmisión de información, (3) desarrollo de sistemas sensoriales y (4) usos químicos y nanobiotecnológicos. En el largo plazo destaca, entre otras áreas, el potencial del autoensamblaje de la materia (fabricación de autoensambladores o nanofábricas y nanorrobots -si es que eso es técnica y físicamente posible).

La descripción más adecuada de nanotecnología tal vez sea la contenida en las definiciones oficiales y que, en general, coinciden en que 
se trata de una tecnología que opera a la nano escala, es decir que trabaja en dimensiones de entre $10^{-6}$ a $10^{-9}$ de metro o, siendo más precisos, la que, como indica la Royal Society, opera manipulando estructuras y sus interacciones de entre los 100 nanómetros (nm) hasta el tamaño de los átomos (aproximadamente $0,2 \mathrm{~nm}$ ). Ello responde a que a esa (nano) escala las propiedades de los materiales pueden ser muy diferentes que a la macro escala (The Royal Society 2004). No obstante, algunas disciplinas como la denominada nanofotónica siguen esencialmente haciendo el mismo tipo de investigaciones que cuando se denominaba fotónica. El caso es compartido por otras áreas de conocimiento científico-tecnológico, lo que sugiere que dicha tendencia de disciplinas madres e hijas (nano) es reflejo de una debilidad de las fronteras entre las disciplinas que operan a una misma escala, pero no como producto de una convergencia espontánea, sino como consecuencia del avance del conjunto de fuerzas productivas capitalistas de fines del siglo XX y de lo que va del XXI.

Por el momento, casi todas las aplicaciones vienen girando en torno al perfeccionamiento de materiales existentes y a la innovación de nuevos materiales. Ésos están siendo utilizados en productos de lujo como bolas de tenis, golf o boliche (a fin de reducir el número de giros que dan las mismas); nanopartículas de zinc para la fabricación de neumáticos de alto rendimiento; fibras para la fabricación de telas con propiedades antimanchas o antiarrugas; nanopartículas para cosméticos, fármacos y nuevos tratamientos terapéuticos; filtros-membranas de agua nanoestructurados y "remedios" ambientales; mejora de procesos productivos mediante la introducción de materiales más resistentes o eficientes; diseño de nuevos materiales para usos que van desde la electrónica, la aeronáutica y toda la industria del transporte hasta su aplicación en armas más sofisticadas y novedosas (explosivos, balística, materiales antibala y stealth, etcétera).

Las propiedades de tales materiales nanoestructurados, sustento de ésas y otras aplicaciones, ya han generado un doble llamado de atención. Por un lado, se observa los amplios beneficios que posibilitaría la reestructuración de prácticamente todo el entorno material que nos rodea pero, por otro lado, se identifica las posibles implicaciones que esa transformación generaría en el ambiente y, de ahí, en la salud, puesto que 
estarían presentes novedosas nanopartículas y nanoestructuras diseñadas por el ser humano (en adelante simplemente denominadas nanopartículas o nanoestructuras) cuyas características, en su gran mayoría, son todavía desconocidas.

\section{PRINCIPALES TIPOS DE NANOESTRUCTURAS Y POTENCIALES RIESGOS AMBIENTALES}

Cuatro son las principales clases en las que en general son clasificadas las nanoestructuras:

a) los materiales de base de carbón: con formas esféricas, elipsoidales o tubulares. Los fullerenos ${ }^{1}$ esféricos son a veces denominados como buckyballs, mientras que los cilíndrico-tubulares como nanotubos (mono o multicapa). Sus propiedades fundamentales son su reducido peso y su mayor dureza, elasticidad y conductibilidad eléctrica, entre otras.

b) los materiales de base metálica: pueden ser quantum dots (puntos cuánticos o transistores de un solo electrón $)^{2}$ o nanopartículas de oro, plata o compuestos de metales reactivos como el dióxido de titanio, entre otras.

c) los dendrímeros: son polímeros nanométricos construidos a modo de árbol en el que las ramas crecen a partir de otras y así sucesivamente. Las terminaciones de cada cadena de ramas pueden diseñarse para ejecutar funciones químicas específicas - una propiedad útil para los procesos catalíticos. Además, dado que tienen cavidades internas, su uso médico para la 'entrega de droga' es factible. Lo mismo sucede con el caso de algunas estructuras de carbón y metálicas.

d) los composites: combinan ciertas nanopartículas con otras o con materiales de mayor dimensión (el caso de arcillas nanoestructuradas es un ejemplo de uso extendido para la fabricación de diversos productos -e.g. autopartes). 
Se ha señalado que las propias propiedades que se están aprovechando de las arriba delineadas nanoestructuras y nanomateriales (por ejemplo su superficie altamente reactiva y su habilidad de atravesar membranas) podrían resultar en peligros importantes, sobre todo en tanto a su potencial grado de elevada toxicidad. ${ }^{3}$

Las implicaciones al medio ambiente en relación a tal toxicidad y a la biodegradabilidad de las nanoestructuras y los efectos de éstas a la salud de la diversidad de especies (incluyendo la humana) tanto en el corto como en el mediano-largo plazo son de consideración puesto que se estima que podrían interferir en las funciones vitales. ${ }^{4}$ La bioacumulación y persistencia de las nanoparticulas a lo largo de la cadena alimenticia es también un factor a observar detenidamente. ${ }^{5}$

El asunto es complejo dados los vacíos en el conocimiento de la nanociencia y la nanotecnología $(\mathrm{NyN})$. Entre ésos, se identifican como de los más importantes: a) la insuficiente definición "del punto" en el que de hecho las propiedades cambian en relación con el tamaño (macro/micro/ nano escala), b) la limitada claridad acerca de esas propiedades de la nanoescala, c) y el casi nulo conocimiento sobre las implicaciones de la interacción de tales o cuales nanoestructuras con el medio natural.

La ingeniera ambiental Nora Savage de la Environmental Protection Agency (Estados Unidos - EUA) indica al respecto:

...los compuestos sobre los que tenemos datos toxicológicos, de destino/transporte o de bioacumulación/biodisponibilidad tal vez necesiten ser re-analizados debido al hecho de que en la nanoescala, las propiedades químicas y físicas usualmente se alteran [...] estamos aprendiendo que 1) estos materiales necesitan ser [física y químicamente] bien caracterizados de modo que los resultados de las investigaciones puedan ser comparables; 2) que puede no ser tan apropiado examinar el uso de nanomateriales diseñados que si se hace desde los productos consumibles en los que éstos son incorporados; y 3) que no es suficiente afirmar que los nanomateriales fijados $o$ incrustados en matrices no implican peligro ambiental o humano alguno - el destino final del producto debe ser 
considerado, si el producto es quemado, puesto en la tierra con líquidos y gases reactivos, reciclado, etc. ${ }^{6}$

Según notifica Roco (del Consejo Nacional de Ciencia y Tecnología de EUA) en la coyuntura actual, las preocupaciones inmediatas cubren entonces aspectos que van desde un mayor conocimiento acerca de la toxicidad de los nano-productos; los mecanismos y rutas de exposición a nanoproductos en el aire, agua y suelo; hasta la efectividad de equipo protector para los que manejan tales productos como ropa y respiradores; las reacciones celulares ante nanopartículas o nanoestructuras, y los mecanismos de prevención de escape de nanopartículas sintéticas en el medio ambiente. ${ }^{7}$

Para ello, es básico determinar, de modo general y particular, las características de los nanomateriales en interacción con el medio natural (y consecuentemente de sus posibles transformaciones). Esto es, por tanto, aspectos como:

- cuáles son sus mecanismos de transporte o movimiento en aire, tierra y agua y cuál es el grado de su capacidad de difusión (e.g. aerodinámica; de filtración en medios porosos como la tierra; o de disolución/dispersión en medios acuosos), de aglomeración, de deposición húmeda y seca;

- de sus propiedades gravitacionales; de su reactividad con moléculas o nanopartículas naturales (no diseñadas o nanoestructuradas) y cómo ello afecta sus características generales, incluyendo su toxicidad; o

- de cambios inducidos por reacciones fotocatalizadoras o inducidas por condiciones anaeróbias. ${ }^{8}$

Ahora bien, lo interesante es que en pleno ascenso del estímulo a la nanotecnología y de los numerosos reconocimientos acerca de los potenciales peligros de las nanopartículas/nanoestructuras por parte de actores gubernamentales ${ }^{9}$, empresariales ${ }^{10} \mathrm{o}$ de $\mathrm{ONG}^{11}$, el financiamiento y por tanto el número de estudios sobre los efectos toxicológicos y medioambientales sean comparativamente aún limitados, aunque al parecer se trata de una situación que está cambiando. 
De cualquier manera, de las indagaciones al respecto, ya se visualizan ciertas características de los nanomateriales que podrían tener implicaciones medioambientales y a la salud. La Royal Society suscribía en 2004 que,

...la evidencia sugiere que por lo menos algunas nanopartículas manufacturadas serán más tóxicas por unidad de masa que aquellas del mismo químico pero de mayor dimensión. Esa toxicidad está relacionada al área de superficie de las nanopartículas (que es mayor respecto a su masa que en el caso de partículas más grandes) y a la reactividad química de la superficie (que puede incrementarse o reducirse mediante el uso de una película envolvente dada - surface coatings). ${ }^{12}$

Tal reactividad química de la superficie de las nanopartículas es de mayor consideración ambiental pues se considera que las enzimas naturales presentes en el medio ambiente pueden cambiar las propiedades de la superficie de las nanopartículas nanoestructuradas y convertirlas en coloides (partículas que no se conglomeran y que no se depositan, por lo que mantienen un alto grado de mobilidad en líquidos). ${ }^{13}$ Estas nanopartículas con características coloidales, según Colvin, podrían ser ideales para la transportación a larga distancia (dígase acuíferos) de material tóxico como contaminantes hidrofóbicos y metales pesados, por ejemplo al reaccionar con moléculas mayores pero de menor movilidad como las ya contenidas en fertilizantes y pesticidas. ${ }^{14}$

Igualmente se advierte que, considerando la reactividad propia de los fullerenos, éstos se tornan potencialmente tóxicos sobre todo si se toma en cuenta que son materiales lipofílicos que tienden a ser almacenados por los organismos en zonas de tejidos grasos. De ahí que Eva Oberdorster ${ }^{15}$ haya corroborado que los fullerenos como el $\mathrm{C}_{60}$ pueden inducir un estrés oxidante en los cerebros de los peces robalo. Y puntualiza: “...no sabemos si los fullerenos son tóxicos porque son metabolizados o si son menos tóxicos debido al metabolismo." ${ }^{16}$

Más aún Lovern y Klaper $^{17}$ sugieren un considerable grado de mortalidad del Daphnia Magna (un diminuto crustáceo, popular alimento para peces de acuario, y usualmente utilizado por su sensibilidad en estudios 
de ecotoxicológicos) cuando son expuestos a nanopartículas de dióxido de titanio (TiO2) y al fullereno $\mathrm{C}_{60}$.

Günter Oberdorster et a ${ }^{18}$ sostienen también que, en base a estudios con ratas, las nanopartículas de carbón pueden entrar directamente desde la nariz hacia los lóbulos olfativos y hasta el cerebro a través de los nervios olfativos. Otras publicaciones han señalado que, además de que ciertos nanomateriales podrían ser efectivos como agentes bactericidas tanto para bacterias positivas como negativas en un cultivo dado, en particular los fullerenos del tipo $\mathrm{C}_{60}$ podrían potencialmente inhibir de modo importante el crecimiento y respiración de los microbios. ${ }^{19}$

Asimismo, Yang y Watts ${ }^{20}$,reportan que las nanopartículas de aluminio (de 13 nanometros) pueden estar involucradas en el enlentecimiento del crecimiento de las raíces de plantas como el maíz, el calabacín, la soya, la col y la zanahoria. Aunque, si las nanopartículas son cubiertas de fenantrene (un hidrocarburo aromático), dichos efectos se "aminoran". En el caso de nanopartículas de aluminia de mayor dimensión no se registró tal impacto en las plantas. Y si bien tales resultados se limitan a ensayos de laboratorio, la incertidumbre sobre sus posibles impactos ya está presente.

De cara a tales y otras indagaciones científicas que de entrada muestran lo limitado que aún es el conocimiento sobre el comportamiento y características de las nanoestructuras, la respuesta general de esa comunidad ha sido que los resultados son provisionales y que traen más preguntas que respuestas, razón por la cual, a decir de Eva Oberdörster, se trata en el peor de los casos más bien de "luces amarillas" y de ninguna manera de "luces rojas”. ${ }^{21}$ De cualquier modo, como acertadamente advierte Altmann,

...llama la atención el hecho de que las nanopartículas estén siendo usadas en productos haciendo sólo uso de la licencia sobre el material en bruto a pesar de que cada publicación sobre la temática sostiene que 1) a la nanoescala la materia muestra diferentes propiedades, y 2) las nanopartículas pueden entrar a través de los poros donde otras más grandes no pueden. ${ }^{22}$ 
Efectivamente, todo indica que los cuidados, las regulaciones y las responsabilidades de quienes están desarrollando y/o estimulando tales o cuales aplicaciones, no están correspondiendo a la complejidad de este frente tecnológico. Como advierte ETC Group desde 2004, “...la nanociencia y la nanotecnología se están desarrollando en un vacío de regulación". ${ }^{23} \mathrm{El}$ señalamiento aún es válido dos años después y a seis años de que se comenzaran a visualizar científicamente los primeros peligros potenciales, aunque hay que reconocer que a partir de 2005 sí se admite formal y extendidamente la necesidad de más investigaciones desde las cuales eventualmente se desprenderían las regulaciones "necesarias".

No sorprende entonces que en este panorama todavía de ausencia de regulación, ya existan cientos de "nano-productos" en el mercado mundial sin mayor regulación específica.

\section{Las nanopartículas y la salud}

El uso de nanoestructuras y nanodispositivos para nuevos sistemas de prevención, diagnostico, tratamiento y regeneración, conforma el corazón de la denominada NanoMedicina -al menos por el momento. Su utilidad, se indica, se debe a que ésos pueden interactuar de modo directo con las biomoléculas, tanto de la superficie de la célula como dentro de ésta.

El espectro de aplicaciones incluye la mejora del intrumental de última generación para una ultra-avanzada resolución espacial, molecular y de sensitividad (nanoimagen ${ }^{24}$; perfeccionamiento y desarrollo de los procesos de diagnóstico in-vivo e in-vitro (e.g. mediante el desarrollo de sofisticados sistemas integrados de nano y micro biosensores ${ }^{25}$ - externos e internos - para el monitoreo tanto en etapas de diagnosis, tratamiento y posterapia; mejoras en los dispositivos laboratorio-en-un-chip $p^{26}$; desarrollo de nanopartículas útiles para su monitoreo dentro del cuerpo o como agentes contrastantes para la obtención de imágenes de mayor resolución); aplicaciones para novedosas formas de 'entrega de fármacos' (más efectivas, focalizadas, de bajo coste y/o diseñadas específicamente para nuevos fármacos) $)^{27}$; tratamientos innovadores terapéuticos y de regeneración de tejidos o hueso (nanobiomimetics); e incluso, implantes de materiales biológico-inertes "mínimamente invasivos". ${ }^{28}$ 
De modo similar a las promesas de la biotecnología, enfermedades como el sida, el cáncer, la diabetes, la osteoartrítis o desordenes degenerativos neurológicos (e.g. Alzheimer, Parkinson, multiesclerosis), cardiovasculares y del sistema nervioso, destacan en las primeras posiciones de la lista de beneficios de la NanoMedicina. ${ }^{29}$ Siguen cuestiones como incapacidades fisiológicas que se intentan resolver en el largo plazo a través de sofisticados implantes que inclusive llegarían a "conectar" biochips al organismo (e.g. implantes de biochips directos al nervio ocular para la generación de imágenes, restitución de células neuronales con biochips, prótesis robotizadas, etcétera).

Sin embargo, los potenciales riesgos a la salud humana son considerables, no sólo como producto de la antes descrita exposición a nanoestructuras vertidas en el medio ambiente y moviéndose a lo largo de la cadena alimenticia, sino también debido a su uso en la NanoMedicina. Así es plenamente reconocido por la Plataforma Europea de Tecnología en NanoMedicina elaborada por representantes del sector industrial (e.g. Bayer, Novartis, Schering, GlaxoSmithKline ${ }^{30}$ ) cuando notaban que:

...los riesgos y los retos de la NanoMedicina comprende asuntos como la toxicidad y la cancerogenecidad, así como la estabilidad a largo plazo y las rutas de excreción de las nanoestructuras artificiales y los retos en la manufactura molecular, la garantía de la calidad y, eventualmente, de la programabilidad de los nanodispositivos. ${ }^{31}$

Por el momento, las implicaciones inmediatas giran entorno a los riesgos de las nanopartículas a la salud humana; de ahí que el grueso de los estudios de riesgo -la mayoría derivados de las propias investigaciones sobre las aplicaciones nanotecnológicas- se avoquen a cuestiones de toxicología de las nanopartículas sea por exposición respiratoria, cutánea 0 por ingestión.

\section{La toxicidad de las nanopartículas inhaladas}

Los especialistas coinciden en que la vía más fácil de penetración de las nanoestructuras es la respiratoria. Dos son las razones centrales. Por un lado, porque, como indican Hoet et $\mathrm{al}^{32}$, las nanopartículas menores a 10 
micrones de diámetro pueden ser inhaladas y, por el otro lado, puesto que mientras más pequeñas, más profundo entran en los pulmones. Aquellas nanopartículas de menos de $100 \mathrm{~nm}$ pueden entrar inclusive a la región alveolar donde se realizan los intercambios de gases a tan sólo 0.5 micrones del torrente sanguíneo. Dadas sus dimensiones, muchas de éstas no son expulsadas sino que se quedan en los tejidos donde el sistema inmunológico procura eliminarlas. Dependiendo de las características físico-quimicas de las nanopartículas, el proceso de eliminación -o fagocitocis- puede ser afectado.

A pesar de que el National Institute for Occupational Safety and Health de EUA señale que la inhalación de nanotubos presentes en el aire solo puede ser mínima, sus implicaciones bien pueden ser importantes. ${ }^{33}$ Un análisis de Warheit y Lam ha demostrado que una vez inoculados los nanotubos de carbono monocapa de modo intratraqueal, éstos generan la formación de granulomas e inflamación intersticial, factores que los hacen mucho más tóxicos que el polvo ultrafino de carbón o de cuarzo. ${ }^{34}$ De ahí que Hoet et $\mathrm{al}^{35}$ sugieran que para determinar con mayor precisión el nivel máximo de tolerancia a una determinada nanoestructura, tal vez sean más importantes las características específicas de la superficie (en particular la reactividad química y las cargas generadas por el contacto de la nanoestructura con la membrana de las células) que el total de su masa. $\mathrm{Y}$ es que nótese que la nanotoxicología busca determinar el espectro o rangos de dosis de las nanoestructuras en el que resulten tolerables por el cuerpo humano para desde ahí establecer la viabilidad de su uso. La cuestión es delicada pues resulta verdaderamente complejo determinar lo que se puede denominar como el "umbral de seguridad" de exposición a tal o cual nanoestructura, y más aún, en su caso, de controlarlo en un contexto en el que ésas y otras nanoestructuras están libres en el medio ambiente.

En términos de salud, la mencionada toxicidad de las partículas (nano/ ultrafinas/finas) se refleja en impactos de malfuncionamiento en el sistema cardiovascular (e.g. arritmia, coagulación) y en posibles afectaciones al sistema nervioso autonómico. ${ }^{36}$ Ello sugiere que hay una traslocación de las partículas, en un grado u otro, desde los pulmones hacia el sistema de circulación (e.g. a través del propio proceso de fagocitosis o endocitosis). ${ }^{37}$ 
La interrogante es cuál es el grado de la toxicidad de las nanopartículas una vez que son traslocadas, sea por la vía respiratoria, cutánea o digestiva (por esta vía solamente las pequeñas nanopartículas pueden traslocarse). ${ }^{38}$

Debe pues observarse cuidadosamente el uso de nanopartículas y dendrímeros para la entrega de fármacos por la vía de su inhalación, una modalidad -la respiratoria- que actualmente se utiliza en el $30 \%$ del total de fármacos a nivel mundial. ${ }^{39}$

Además, no ha de dejarse de lado las consecuencias a la salud de potenciales escenarios de exposición permanente. Algunos estudios al respecto ya estiman que la exposición crónica a nanoestructuras como el $\mathrm{TiO}_{2}$-dióxido de titanio- puede incluso generar tumores cancerígenos de pulmón. ${ }^{40}$ En paralelo tómese nota del trabajo de Yusaku Nakabeppu et $\mathrm{al}^{41}$, que sugiere que los agentes medioambientales con capacidad de causar cambios químicos en el ADN pueden incrementar a lo largo de la vida la cantidad de mutaciones que ocurren en nuestro ADN durante la formación de huevos o células espermatozoides y por tanto, heredar tales mutaciones que podrían influir fuertemente en el reordenamiento de los cromosomas. Asimismo, adviertase la posibilidad de que las nanopartículas que puedan atravezar las membranas celulares, potencialmente interfirirían en el funcionamiento de la misma, incluyendo el proceso de transcripción del ADN.

\section{Los cosméticos y los potenciales riesgos de sus nanopartículas. La exposición cutánea.}

La piel es una barrera de unos 10 micrones compuesta de tres capas: la epidermis (células muertas fuertemente queratenizadas), la dermis y el tejido graso subcutáneo.

Aunque se cree que el grueso de partículas no logran atravesar siquiera la epidermis (tanto por factores exógenos como endógenos ${ }^{42}$ ), hay señalamientos acerca de que las nanopartículas de menor dimensión potencialmente podrían atravesarla y llegar al sistema circulatorio.

No obstante el desacuerdo científico es mayor pues se dice que las micropartículas de dióxido de titanio empleadas en los bloqueadores solares de rayos UV logran atravesar la epidermis e incluso llegar al fondo 
de los folículos de pelo, pero ello no es considerado como penetración por autores como Lademann et $a^{\mu 3}$. En contracorriente, Kreilgaard ${ }^{44}$ sostiene que una fracción muy pequeña de micropartículas de dióxido de titanio sí logran penetrar la piel e interactuar con el sistema inmunológico. Tinkle et $a^{45}$ igualmente han demostrado que partículas de 0.5 a un micrón pueden ocasionalmente alcanzar la dermis e interactuar con el sistema inmunocirculatorio.

Sin embargo, de lo que están hablando esos autores es de micropartículas de un solo material, no de nanopartículas de diversa naturaleza. Esta segunda dimensión de la discusión es aún más intensa. Para Hoet et al, es más factible que las nanopartículas penetren la piel que las micropartículas, al tiempo que consideran que dependiendo del material éstas pueden ir a capas más profundas aunque, no hay por el momento contundentes indicaciones de que puedan entrar al sistema circulatorio; a menos que la piel esté dañada de antemano (e.g. acné, heridas, quemaduras, etcetera). ${ }^{46}$

Según Colvin, en el caso de nanopartículas de titanio y de óxido de zinc (también usadas en bloqueadores solares) se estima que aunque aquellas mayores de $40 \mathrm{~nm}$ difícilmente penetrarían la epidermis, dada su reactividad fotocatalizadora es posible que degraden la propias fórmulas de los bloqueadores una vez expuestas a la luz. Tal proceso, aseguran diversos análisis basados en micropartículas de titanio, daña las moléculas biológicas pero el efecto no es tan grave en comparación a la exposición solar. Sin embargo, los estudios para partículas menores de 100 nm no existen. ${ }^{47}$ Aún así, Advaced Nanotechnology (Australia) y Oxonica (Inglaterra) sostienen que sus productos son fotoestables (o que no producen radicales libres cuando son expuestos a la luz). ${ }^{48}$

En medio de este controversial escenario en el que los resultados se contradicen, son inconclusos o incluso en el que aún no hay investigación, Paul Borm del Center of Expertise in Life Sciences (Holanda) sostiene:

...estamos tratando de encontrar una explicación de por qué pequeñas cantidades de partículas...pueden tener esos tremendos efectos. La gente [los investigadores] está tratando de encontrar la bala mágica. Algunos dicen que son 
los metales en las partículas, otros que son los agentes y un enorme grupo dice que son las nanopartículas en sí mismas. Esto último es una atractiva teoría, pero se necesita mayor investigación. ${ }^{49}$

En el caso concreto de los potenciales riesgos del uso de nanoestructuras en cosméticos, el proyecto NanoDerm, financiado desde el Research and Technology Development Programme de la Comisión Europea, sugiere colocarse en dicha dirección, pero hasta mediados de 2006 ni siquiera se había dado a conocer el más mínimo avance sobre sus actividades. ${ }^{50}$

Lo llamativo entonces es que ya se encuentran en el mercado numerosos productos de cosmética que hacen uso de nanopartículas. Según el Environmental Working Group (EUA), de los cerca de 10,500 ingredientes que se utilizan en la industria de los cosméticos, tan sólo el $11 \%$ ha sido estudiado por paneles de expertos de la propia industria. ${ }^{51}$ En lo que respecta a nanoestructuras, no se sabe con exactitud cuantos ingredientes realmente las contienen y cuantos más bien tienen micromoléculas. Tampoco queda del todo clara la consistencia de su uso, aunque L'Oreal parece colocarse a la vanguardia con docenas de nanosomas patentados y un gasto en nanotecnología de alrededor de unos 500 millones de euros. ${ }^{52}$ Pero ciertamente no es la única.

Una revisión de los productos que están en el mercado y que abiertamente declaran hacer uso de nanoestructuras realizada, por un lado, por el Woodrow Wilson Internacional Center for Scholars ${ }^{53}$ y , por otro lado, por Friends of the Earth ${ }^{54}$, indica que entre los cosméticos se incluyen marcas, además de L’Oreal (nanosomas), a: Chanel, Johnson \& Jonson, Procter \& Gamble, Revlon, Lancome, Clinique (nanopartículas/ nanocápsulas); Eternalis, Hawaiian Tropic y NuCelle (nanopartículas); Zelens (buckyballs); BioNova Cosmetics (nano y pico partículas), entre muchas otras. ${ }^{55}$

El debate fuera de la esfera de los impulsores de la nanotecnología es realmente tenso ya que, en este caso, la comunidad de la industria de los cosméticos sostiene que sus productos son seguros (aunque no revelan los resultados de ningún estudio que lo avale). Por ejemplo, John Bailey de 
la Cosmetic, Toiletry and Fragance Association (EUA) indicó que aunque aún son pocos los productos que usan nanopartículas, “...desde luego dentro de las leyes de cosméticos ya hay suficientes revisiones y balances que asegurarán que los productos y sus ingredientes sean seguros. ${ }^{~} 56$

No obstante, es sabido que la Food and Drug Administration (EUA) generalmente solo investiga los cosméticos si surgen cuestionamientos sobre su seguridad una vez que ya están en el mercado puesto que no hay ningún proceso de aprobación previo a la comercialización de fórmulas de cosméticos. ${ }^{57}$ Tampoco existe la regulación en ningún país del mundo en el que se exija el etiquetamiento de los productos que contengan nanoestructuras. ${ }^{58}$

En palabras del premio Nobel en química (1996) Robert Curl, quien co-descubriera los fullerenos:

... yo tomaría un camino conservador tratando de evitar usar los cosméticos. Mientras tanto, hay que conservar el juicio en los verdaderos méritos o desméritos de su uso. ${ }^{59}$

Consecuentemente, ocho grupos entre los que están Friends of the Earth y el The International Center for Technology Assessment presionaron en mayo de 2006 para un fortalecimiento de las regulaciones estadounidenses entorno a los cosméticos que incluyen nanoestructuras. ${ }^{60}$ El llamado a ser precavidos, independientemente de su modalidad (e.g. revisión-regulación, moratoria, prohibición), sin duda debería ser extendido a nivel internacional pues se corre el riesgo de caer en lo que se califica como "una lección tardía de alerta temprana"). ${ }^{61}$

Aunque, la Comisión Europea inicialmente aseguraba en 2004 movida por un optimismo tecnológico poco meditado, que:

...los riesgos parecen ser controlables...sabemos ya que algunas nanopartículas no son dañinas a la salud. Por eso se utilizan en las cremas de protección solar como factor de protección contra la luz o se mezclan con otros materiales a los que van enlazadas de manera que el usuario no siquiera entra en contacto con ellas. La industria también está aplicando las medidas de seguridad 
adecuadas para evitar cualquier riesgo sanitario a sus clientes o empleados. ${ }^{62}$

Los estudios o regulaciones a las que pudiese estarse refiriendo la Comisión hasta la fecha son públicamente desconocidos.

\section{Nano-agroindustría: nanoestructuras en su estómago}

Con pocas aplicaciones en el mercado, las ganancias "nano" del sector se estiman en 2.6 mmdd para 2003 y en 7 mmdd para 2006. Las proyecciones sugieren hasta $20 \mathrm{mmdd}$ al cierre de esta década. Tal boom se debe a que la nanotecnología promete impactar e incluso revolucionar la agroindustria como un todo. Es decir, en tanto a la producción agrícola, el procesamiento de alimentos y su empaquetamiento.

En lo que respecta a la producción agrícola, se habla de "cultivos de precisión", sobre todo de bienes relativamente caros y que por tanto permiten fuertes inversiones. Dígase, por ejemplo, la uva para vino o el tomate cherry de cultivo hidropónico. El paquete nanotecnológico incluye, además del eventual "perfeccionamiento" de la manipulación a nivel atómico-molecular del ADN, el uso conjunto de computadoras, sistemas de posicionamiento global, micro/nano dispositivos sensoriales remotos, así como nuevos agroquímicos "nanomejorados"; todo con el objeto de: a) monitorear en tiempo real las condiciones ambientales y del suelo, así como del desarrollo de las plantaciones (incluyendo el estrés); b) controlar los insumos empleados; e c) identificar eventuales patógenos, plagas $\mathfrak{u}$ otros inconvenientes como los relacionados a las condiciones óptimas de almacenamiento de granos u otros productos agrícolas (e.g., humedad, temperatura, etc).

$Y$ es que se considera posible un "tratamiento inteligente" que, similar al de la nanomedicina, pueda monitorear y diagnosticar la salud de los cultivos y, consecuentemente, a partir de desarrollar "nanoestructuras inteligentes", entregar dosis adecuadas de herbicidas, pesticidas, nutrientes, etcétera. De modo similar funcionaría la nanoveterinaría tanto en lo que refiere al monitoreo, diagnosis, tratamiento e intervención terapéutica de animales. Monsanto (EUA), Syngenta (Suiza), Bayer y BASF (Alemania) se perfilan a la cabeza del negocio. 
Syngenta (Suiza), por ejemplo, ya está usando nanoemulsiones en sus productos. Tal es el caso de su regulador de crecimiento Primo $M A X X^{63}$ o algunos de sus insecticidas para el control de pestes del algodón, arroz, cacahuates y soya, cuyas nanopartículas que contienen el químico activo pueden romperse al contacto con las hojas o con medios alcalinos como el estómago de ciertos insectos (patente estadounidense $6544540)$.

Enlocorrespondiente a los alimentos procesados las nanoaplicaciones son diversas aunque el grueso giran entorno al uso de diversas nanoestructuras como plataformas ideales para el nanodiseño, introducción y funcionalidad de conservadores, saborizantes, nutriceúticals (vitaminas, etc) y otros aditivos para la elaboración de alimentos "a la medida" del consumidor. El sostén tecnológico es el diseño de nanoestructuras que puedan ser "activadas" al contacto con la saliva o los jugos gástricos, entre otros medios. En el primer caso se habla de intensificadores de sabor, mientras que en el segundo, de bloqueadores del mismo. El pan de caja de la autraliana Tip Top ya hace uso de nanopartículas rellenas de aceite de pescado como fuente de Omega 3 , mismas que se rompen una vez ingeridas de modo que el consumidor no detecte el "mal sabor".

Las expectativas han llevado al grueso de los gigantes de la industria a desarrollar todo tipo de nanoestructuras multi-funcionales, muchas de las cuales tendrán a su estómago como destino. Tal es el caso de Nestlé (dueña también del 49\% de L’Oreal; en la vanguardia de nanocosméticos), Kraft (EUA; que encabeza la iniciativa privada involucrada en el diseño del plan de acción de ese país en nanoalimentos), Heinz (EUA), Unilever (Reino Unido/Holanda), entre otras.

Las nanoaplicaciones en empaquetamiento son revolucionadoras. De las investigaciones más sonadas está la "lengua electrónica" de Kraft, una plataforma de nanosensores extremadamente sensibles a gases que desprenden los alimentos cuando se hechan a perder y que hace que un indicador cambie de color. Se suman materiales como la pélicula de embalaje Durethan (de Bayer Polymers) que es más fuerte y resistente al calor que el resto de las que se encuentran en el mercado. Además, protege los alimentos envasados de la humedad y del oxígeno por la vía de 
nanopartículas de silicate introducidas a la película nanoestructurada. El uso de nanopartículas bloqueadoras de rayos UV (de dióxido de titanio), antimicrobianas y antibacterianas (i.e., de plata) también prometen alargar el tiempo de vida de productos perecederos.

Se suma el "empaquetamiento inteligente" y que incluye técnicas de "nano-impresión” (e.g. nanocódigos de barra) y de nanocodificación de materiales plásticos y de papel para propósitos de autentificación por radio frecuencia. Se trata de razgos útiles tanto para la protección de marcas y patentes, como para el rápido y fácil manejo de millones de paquetes por parte de cadenas como Walmart (EUA) o Carrefour (Francia).

\section{CONSIDERACIONES FINALES}

Conviene preguntarse, hasta qué punto vale la pena correr los riesgos de abrir una potencial caja de Pandora de la nanotecnología y a qué coste (actual y futuro); sobre todo en un panorama en el que buena parte de la investigación, a decir de la Dra. Dehmer, de la Office of Basic Energy Sciences del Departamento de Energía de EUA, está centrándose en un gran reto:

...tomar diferentes tipos de nanoestructuras en las que la naturaleza no ha pensado, ponerlas juntas en diversas formas de modo que podamos hacer cosas que la naturaleza no ha hecho y, en particular, hacer cosas que sean más robustas que los sistemas naturales. ${ }^{64}$

Y es que la potencial y eventual "minimización" de los efectos de una tecnología dada en el largo plazo -por la vía del propio desarrollo tecnológico-, no parece ser conveniente pues las consecuencias de nuestras decisiones actuales marcarán y tendrán que ser resueltas (o no) por las generaciones futuras, si es que nuestros cálculos sobre el avance tecnológico fueron correctos y si nuestra dimensión de precaución sobre la amplitud de los efectos fue "adecuada".

Sarewitz y Woodhouse observan que,

...resulta una sorprendente ironía de la innovación tecnológica que a pesar de aplicar toda nuestra inteligencia 
racional al corto plazo, debamos confiar nuestro bienestar de largo plazo a la esperanza de que las buenas consecuencias imprevistas de nuestras invenciones contrapesen las malas. En la medida en que la potencia de nuestras tecnologías continúa acelerándose, esto parece más que imprudente. ${ }^{65}$

Así pues, en el caso de las nanoestructuras se han de realizar estudios sobre su reactividad per se y sus interacciones con el medio ambiente, no sólo inmediatas y reducidas a espacios determinados, sino también en el largo plazo y en una situación de saturación ambiental global con diversas nanopartículas pululando y potencialmente reactivas. Lo mismo, sucede en relación a la salud humana pues la investigación preventiva no sólo debe enmarcarse entorno a análisis nanotoxicológicos (exposición inmediata) sino a las potenciales implicaciones de largo plazo que se podrían presentar por una convivencia permanente con esas nanoestructuras (dígase potenciales modificaciones genéticas hereditarias). En este contexto de incertidumbre a lo largo de toda la línea del tiempo, se ha hecho un llamado para regular, caso por caso, el uso de nanoestructuras en alimentos y embalajes ${ }^{66}$; aún cuando las mismas partículas de mayores dimensiones ya estén aprobadas por los entes gubernamentales responsables. Pero, para ello es imprescindible que fluya la información sobre lo que está sucediendo en los laboratorios y centros de producción (e.g. tipos y cantidades de materiales empleados y desechos generados, etc). Y es que mucha información necesaria para tales estudios de flujos de nanomateriales se considera confidencial bajo criterios como el secreto industrial y, en ciertos casos -como un asunto sensible por sus posibles implicaciones entorno al avance y consolidación de la comercialización de la nanotecnología. ${ }^{67}$ Ello dificulta cualquier intento serio de manejo del riesgo (sea cual fuese éste, e.g. principio precautorio), al tiempo que entorpece la aplicación de un marco regulatorio acorde a la complejidad de las nanotecnologías. No es casual que en este panorama, la industria nanotecnológica y los gobiernos que la apoyan, en EUA en boca de Mihail Roco, prefiera hablar de un "ambiente de regulación a nivel global que se autodefina”. ${ }^{68}$ Es decir, lo que en los hechos viene sucediendo. Por ejemplo, lo laxo del actual contexto de control y regulación en EUA, es patente cuando el grupo asesor en nanotecnología de la FDA se limita 
a proponer a la Administración como "fuente guia para los productores sobre cuando el uso de nano-ingredientes podría (sic) requerir la entrega de información adicional".

$\mathrm{El}$ asunto del manejo social de las implicaciones de la nanotecnología no es cualquier cosa pues la incertidumbre e implicaciones de la generación de una "segunda naturaleza" ${ }^{9}$, sugieren ser de orden mayor. Se trata de un frente tecnológico que si bien es cierto coloca en gran medida las implicaciones futuras en el ámbito de lo incierto, no por ello deja de ser más, o menos, riesgoso.

Por tanto, son imprescindibles revisiones valorativas a fondo que eviten soluciones unidimensionales y tecnológicamente optimistas, al tiempo que se toma nota, detenidamente y en toda su amplitud espaciotemporal, de las particularidades de tales o cuales casos. Así, con este tipo de ejercicios de reflexión se pueden visualizar con más amplitud los posibles costos de las decisiones tomadas hoy; quiénes pagarían ese coste ahora y en las generaciones futuras, y quiénes recibirían tal o cual beneficio (potencial o real). No es más que teniendo clara la complejidad del asunto tanto en el corto como en el mediano y largo plazo que se pueden tomar decisiones más responsables, aunque no necesariamente correctas; y más aún, socialmente concertadas (con la siempre compleja cuestión de cómo tomar en cuenta "las opiniones" de las generaciones futuras).

Por lo pronto, en la coyuntura de toma de decisiones y del inevitable manejo (o no) del riesgo y del peligro e independientemente de la manera en la que éstos sean gestionados (pro-activa y/o reactivamente; ya sea en el "aquí y el ahora” o en el "hoy, aquí, el mañana y allá"), Andrew Maynard sugería a principios de 2006 que los gobiernos y la industria debían incrementar su gasto en investigación sobre los peligros ambientales y a la salud de las nanotecnologías pues de los 9 millárdos de dólares que se gastan a nivel mundial en IyD nanotecnológico, solamente son destinados a ese tipo de investigaciones entre 15 y 40 millones por año. Es suficiente? Cuestionaba Maynard al tiempo que respondía: “...bueno, creo que eso depende de que tan serios somos en cuanto al desarrollo de nanotecnologías seguras."70 


\section{NOTAS}

1 Un fullereno es la tercera forma alotrópica de carbono (diamante, grafito y fullereno). Fue descubierta en 1985 como una sustancia donde cada molécula poseía sesenta átomos de carbono engarzados a modo esférico o elipsoidal. Si bien el C60 es el más común, también hay fullerenos de carbono de más átomos como el C70, C84, C240, C540. En 1991 se detectó una forma más de carbono, el "nanotubo". Un nanotubo es un fullereno muy grande pero en forma lineal.

2 Un punto cuántico es una estructura cristalina a nanoescala que puede transformar la luz. Se puede definir como una partícula de materia tan pequeña [el 70\% de sus átomos son de superficie] que la adición de un único electrón produce cambios en sus propiedades. El punto cuántico se considera que tiene una mayor flexibilidad que otros materiales fluorescentes, lo que lo hace apropiado para utilizarlo en construcciones a nanoescala de aplicaciones computacionales donde la luz es utilizada para procesar la información. Los puntos cuánticos están hechos de una variedad de diferentes componentes, tales como cadmio selenio (Diccionario de Conceptos de Euroresidentes, en: www.euroresidentes.com/futuro/nanotecnologia/diccionario/puntos_ cuanticos.htm).

3 Royal Society. Nanosience and nanotechnologies: opportunities and uncertainties. Reino Unido, Julio de 2004. Op cit: 35 .

4 Ibid: 45.

5 La bioacumulación de nanopartículas es en buena medida determinada por las propiedades de sus superficies, lo que a su vez define si son retenidas por tejidos grasos, hueso o proteínas del cuerpo. La persistencia dependerá en cambio de su descomposición o modificación por el medio ambiente (Ibid: 45).

6 En: Delgado, Gian Carlo. "NanoConceptions: A Sociological Insight of Nanotechnology Conceptions". The Journal of Philosophy, Science \& Law. University of Miami. EUA, 1 de julio de 2006.

7 Roco, Mihail. "Responsable of Nanotechnology." Environmental Science \& Technology. EUA, 1 de marzo de 2005: 108A.

8 EPA - Environmental Protection Agency. Nanotechnology White Paper. EPA's Science Policy Council. EUA, 2 de diciembre de 2005: 35-8.

9 Por ejemplo: 1) Roco, Mihail C., and Bainbridge, William S. Societal Implications of Nanoscience and Nanotechnology. National Science Foundation. EUA, marzo de 2001. 2) Tomellini, Renzo and Roco, Mihail C. Nanotechnology Revolutionary Opportunities and Societal Implications. European Commision. Bélgica, febrero de 2002. 3) EPA - Environmental Protection Agency EPA Nanotechnology and the environment: Applications and Implications. Washington, EUA, agosto de 2002. 4) Roco, Mihail. C, and Bainbridge, William S. Nanotechnology: Societal Implications - Maximazing Benefits For Humanity. National Nanotechnology Initiative. EUA, 3-5 de diciembre de 2003. 5) Royal Society. Julio, 2004. Op cit; 6) Department for Environment, Food and Rural Affairs. Characterising the Potential Risks Posed by Engineered Nanoparticles. UK Government. Londres, 2005.

10 Véase: Hett, Ammabelle. Nanotechnology: Small Matter, Many Unknowns. Swiss Reinsurance Company. Suiza, 2004.

11 Las referencias clásicas son: 1) ETC Group, The Big Down: From Genomes to Atoms. Canadá, enero de 2003; y 2) Arnall, Alexander H. Future Technologies, Today's Choices. Greenpeace Environmental Trust. Londres, julio de 2003.

12 Royal Society, The., julio 2004. Op cit: ix.

13 Por ejemplo, Colvin sostiene que ciertos nanomateriales podrían absorber pequeños contaminantes como el cadmio y otros compuestos orgánicos. Y, consecuentemente como los coloides, podrían ser una vía de transporte rápido y a larga distancia de contaminantes en las aguas subterráneas (Colvin, Vicky. "Responsible Nanotechnology: Looking Beyond the Good News", EurekAlert! Nanotechnology In Context. Noviembre de 2002. Disponible en: www.eurekalert.org/context. php?context=nano\&show=essays 
\&essaydate $=1102$ ).

14 Vicki Colvin así lo asegura a partir de citar el trabajo de su colega Mark Wiesner. Colvin, noviembre de 2002. Op cit. Léase también: Hett, 2004. Op cit.

15 Véase: Oberdorster, E. "Manufactured nanomaterial (fullerenes, C60) induce oxidative stress in the brain of juvenile largemouth bass”, Environmental Health Perspectives. Vol. 12 No. 10. EUA, 2004:1058-1062.

16 Citada en Helland, Asgeir. Nanoparticles: A Closer Look at the Risks to Human Health and the Environment. Internacional Institute for Industrial Environmental Economics. Tesis de Maestria. Suecia, octubre de 2004: 23.

17 Lovern, Sarah B., y Klaper, Rebecca. "Daphinia Magna Mortality When Exponed to Totanium Dioxide and Fullerene (C60) Nanoparticles." Environmental Toxicology and Chemistry. Vol 25. No. 4. EUA, 2006: 1132-1137.

18 Véase: a) Oberdorster, G., Sharp, Z., Atudorei, V., Elder, A., Gelein, R., Kreyling, W., and Cox, C. "Translocation of inhaled ultrafine particles to the brain." Inhalation Toxicology No.16. EUA, 2004: 437445; b) Oberdorster, G., Sharp, Z., Atudorei, V., Elder, A., Gelein, R., Lunts, A. et al. "Extrapulmonary translocation of ultrafine carbon particles following whole-body inhalation exposure of rats." Journal of Toxicology and Environmental Health. A 65 EUA, 2002: 1531-1543.

19 Véase, EPA, 2 de diciembre de 2005. Op cit: 59.

20 Yang, L., y Watts, D.J. "Particle surface characteristics may play an important role in phytotoxicity of alumina nanoparticles.” Toxicology Letters. No.158: 122-132. EUA, agosto de 2005.

21 Feder, Barnaby J. "Health Concerns in Nanotechnology. The New York Times. EUA, 29 de marzo de 2004.

22 Delgado-Ramos, Julio de 2006. Op cit.

23 Palabras de Dr Kathy Jo Wetter. Cita tomada de Delgado-Ramos, 1 de julio de 2006. Véase también dicho señalamiento en: ETC Group, "Nanotech Advancing in Legal Vacuum”, ETC Group News Release. Canadá, 30 de junio de 2004.

24 Por ejemplo nuevas generaciones de microscopios ópticos, de microscopios de efecto túnel, de microscopios electrónicos o de imagen de espectrometría masiva.

25 Un biosensor es un sensor que contiene un elemento biológico -como una enzima- capaz de reconocer o "señalar" (a través de un cambio bioquímico) la presencia, actividad o concetración de una molécula específica en una solución.

26 Inicialmente denominados "microarrays" o "biochips" y ahora de modo más preciso para el caso de las aplicaciones médicas de diagnóstico como "laboratorios-en-un-chip”. Son una herramienta diseñada para el análisis rápido de hasta las diferencias más mínimas en los códigos genéticos o de la presencia de patógenos y/o moléculas en las muestras (e.g. sangre). Para mayores referencias y una lista de las principales empresas involucradas en su desarrollo, véase: Gershon, Diane. "DNA microarrays, More than than gene expression", Nature. Vol. 437. No. 7055. 20 de octubre de 2005: 1195-1200.

27 Especialización en la que se combinan la ciencia de los polímeros, la farmacéutica, la química bioconjugada, la biología molecular y la nanotecnología (ingeniería de nanopartículas). Varias son las nanoestructuras útiles, pero en especial destacan los nanotubos de carbono, los fullerenos y los dendrímeros pues pueden llevar cargas de droga en su interior. El potencial de las nanoestructuras es que no sólo funcionarían como nuevos mecanismos de entrega de fármacos, sino que previa y simultáneamente como medios de diagnóstico.

28 European Commission. European Technology Platform on NanoMedicine: Vision Paper and Basis for a Strategic Research Agenda for NanoMedicine. Bélgica, septiembre de 2005: 7-9.

29 Ibid: 9.

30 Destacan además de las mencionadas, bioMérieux, UCB, Philips Medical Systems, Siemens AG, Neuropharma, ION-TOF GMBH, Genomita, Gambro, Selex Sensors and Airborne Systems, etre otras. Véase lista de expertos en: European Commission, septiembre de 2005. Op cit: 2-3. 
31 Ibid: 12.

32 Hoet, Peter H., Brüske-Hohlfeld, Irene., y Salata Oleg. "Nanoparticles - Known and Unknown Health Risks” Nanobiotechnology. Vol. 2. No. 12. EUA, 2004.

33 Maynard, A.D., Baron, P.A., Foley, M., Shevedova, A.A., Kisin, E.R., Castranova, V. "Exposure to Carbon Nanotube Material: Aerosol Release During Handling of Unrefined Single Wall Carbon Nanotube Material." Journal of Toxicology and Environmental Health. No. 67. EUA, 2004: 87-107. Citado en Hoet et al, 2004. Op cit.

34 1) Warheit, D.B.Laurence, B.R., Reed, K.L., Roach, D.H., Reynolds, G.A., Webb, T.R. “Comparative Pulmonary Toxicity Assessment of Single Wall Carbon Nanotubes in Rats. Toxicological Sciences. No. 77. 2003: 117-125; y 2) Lam, C.W., James, J.T., McCluskey, R., Hunter, R.L., "Pulmonary Toxicity of SingleWall Carbon Nanotubes in Mice 7 and 90 Days alter Intratracheal Instillation”. Toxicological Sciences. No. 77. 2003: 126-134. Citados en Hoet et al, 2004. Op cit.

35 Hoet et al, 2004. Op cit.

36 Véase: a) Yeates, D.B., Mauderly, J.L. "Inhaled environmental/occupational irritants and allergens: mechanisms of cardiovascular and systemic responses: Introduction.” Environmental Health Perspectives. No. 109. 2001: 479-481; b) Gold, D.R., Litonjua, A., Schwartz, J., Lovett, E., Larson, A., Nearing, B., Allen, G., Verrier, M., Cherry, R., Verrier, R. "Ambient pollution and Herat rate variability. Circulation. No. 101. 2000: 1267-1273; c) Liao, D., Creason, J., Shy, C., Williams, R., Watts, R., Zweidinger, R. "Daily variation of particulate air pollution and poor cardiac autonomic control in the elderly. Environmental Health Perspectives. No. 107. 1999: 521-525. Citados en Hoet et al, 2004. Op cit.

37 Hoet et al, 2004. Op cit. Véase también: Oberdörster, G. "Pulmonary effects of inhaled ultrafine particles." Internacional Archives of Occupational and Environmental Health. No. 74. 2001: 1-8.

38 Ya desde 1926 se sabe que las partículas pueden traslocarse desde el lumen hasta el tracto intestinal por la vía de agregaciones o del tejido linfático intestinal. Por el momento se sabe que mientras más pequeñas sean las partículas más rápido podrían permutarse al mocus y alcanzar los entericitos colónicos. Desde ahí, éstas pueden penetrar tanto el tejido linfático como los tubos capilares. Según un estudio de Jani et al, partículas de un diámetro de $14 \mathrm{~nm}$ se permutan en 2 minutos, las de $415 \mathrm{~nm}$ en 30 minutos y las de $1000 \mathrm{~nm}$ no pueden traslocarse (Jani, P., Halbert, G.W., Langridge, J., Florence, A.T. "The uptake and translocation of latex nanospheres and microspheres alter oral administration to rats". Journal of Pharmacy and Pharmacology. No. 41. 1989: 809-812. Citado en Hoet et al, 2004. Op cit).

39 European Commission, septiembre de 2005. Op cit: 25.

40 Heinrich, U., Muhle, H., Hoymann, H.G. Mermelstein, R. "Pulmonary function changes in rats alter chronic and subchronic inhalation exposure to various particulate matter. Experimental Pathology. No. 37. 1989: 248-252; y, Lee, K.P., Nelly, D.P., Scheneider, P.W., Trochimowicz, H.J. "Inhalation toxicity study on rats exponed to titanium tetrachlorine atmosferic hydrolysis products for two years. Toxicology and Applied Pharmacology. No. 83. 1986: 30-45. Citados en Hoet et al, 2004. Op cit.

41 Coghlan, Andy. "How Chemicals Can Speed Up Evolution", New Scientist. EUA, 6 de mayo de 2006: 16.

42 Entre los endógenos vale mencionar la edad de la piel, la constitución anatómica del sitio de aplicación, el control homeostático, etc. Entre los exógenos, enlístese la dosis, el vehículo de aplicación (líquido, sólido), la reactividad protéica o la valencia.

43 Lademann, J., Weigmann, H., Rickmeyer, C., Barthelmes, H. Schaefer, H., Mueller, G., Sterry, W. "Penetration of Titanium dioxide microparticles in a sunscreen formulation into the horny layer and the follicular orifice." Skin Pharmacology and Applied Skin Physiology. No. 12. 1999: 247-256. Citado en Hoet et al, 2004, Op cit.

44 Kreilgaard, M. "Influenceof microemulsions on cutaneous drug delivery. Advance Druge Delivery Reviews. No. 54. 2002: S77-S98. Citado en Hoet et al, 2004. Op cit. 
45 Tinkle, S.S., Antonini, J.M., Rich, B.A., Roberts, J.R., Salmen, R., DePree, K., Adkins, E.J., “Skin as a route of exposure and sensitization in chronic beryllium disease. Environmental Health Perspectives. No. 111. 2003: 1202-08. Citado en Hoet et al, 2004. Op cit.

46 Hoet et al, 2004. Op cit.

47 Colvin, 2003. Op cit: 1167.

48 Véase: www.advancednanotechnology.com; y, Pitman, Simon. "Nanotech UV maker buys up US rival company", Cosmetics Design.com 27 de febrero de 2006. Disponible en: www.cosmeticsdesigneurope.com/news/ng.asp?n=66085-oconica-nanoplex-uv-absorber-optisol. Citados en: Miller, Georgia. Nanomaterials, Sunscreens And Cosmetics: Small Ingredients, Big Risks. Friends of the Earth. Australia/ EUA, mayo de 2006: 12.

49 Citado en Helland, octubre de 2004. Op cit: 21. Por ejemplo, según cita Helland, Eva Oberdörster sostiene que los puntos cuánticos serán altamente tóxicos porque están hechos de metales. Lo mismo sugiere con el caso de los fullerenos que son activos a la reducción-oxidación, diseñados para ser catalizadores (Ibid: 25).

50 Participan la Universidad de Leipzig (Alemania), la Universidad de Bordeaux (Francia), la Universidad de Debrecen (Hungría), La Universidad de Krakovia (Polonia), el Instituto Tecnológico y Nuclear (Portugal) y el Instituto de Física Nuclear de Niewodniczanski (Polonia). Véase: www.uni-leipzig.de/ nanoderm/index. html

51 Véase: www.ewg.org/reports/skindeep2/newsrelease.php

52 Rogers, Lois. "Safety fears over 'nano' anti-ageing cosmetics”, The Sunday Times - Britain. Reino Unido, 17 de julio de 2005.

53 Véase en: www.nanotechproject.org/44/consumer-nanotechnology

54 Miller, mayo de 2006. Op cit.

55 Para una búsqueda de los grados de preocupación de los ingredientes de los cosméticos (nanos o no), vésase la página electrónica “Skin Deep” del Environmental Working Group (EUA): www.ewg.org/reports/ skindeep2/

56 Boyce, Nell. "Safety of Nano-Cosmetics Questioned”, Health \& Science. EUA, 16 de mayo de 2006.

57 Ibid.

58 Miller, mayo de 2006. Op cit: 5.

59 Halford, Bethany. "Fullerene For The Face: Cosmetics containing C60 nanoparticles are entering the market, even if their safety is unclear". Chemical \& Engineering News. Vol. 84. No. 13. EUA, 27 de marzo de 2006: 47. Disponible en: http://pubs.acs.org/cen/science/84/8413sci3.html

60 Lisa Archer de Friends of the Earth era contundente en su declaración a la prensa al señalar que, “...las corporaciones deberían de detener la comercialización de nano productos hasta que esos (nano)materiales sean probados seguros y dejar de amenazar a sus clientes como conejillos de indias. Véase: Pitman, Simon. "Lobby groups question cosmetic nanoingredients", Cosmetics Design.com EUA, 18 de mayo de 2006. Consúltese en: www.cosmeticsdesign.com/news/ng.asp?id=67800

61 Véase: European Commission. Lecciones tardías de alertas tempranas: el principio precautorio, 1896-2000. European Environment Agency. Bélgica, 2001**. Friends of the Earth lo expresa así: “...The early warning signs surrounding nanotoxicity are serious and warrant a precautionary approach to the commercialization of all products containing nanomaterials" (Miller, mayo de 2006: 17).

62 European Commission, La nanotecnología. Innovaciones para el mundo del mañana. Bélgica, 2004*: 47.

63 Véase: www.syngentaprofessionalproducts.com/to/prod/primo/

64 Palabras de la Dr. Patricia M. Dehemer del Departamento de Energía ante una audiencia del Senado de EUA. Véase: US Senate. Roundtable on Health Technology. Hearing of the Committee on Health, Education, Labor, and Pensions. No. 108-247. US Government Printing Office. EUA, 23 de Septiembre de 2003: 7. Tómese nota entonces de la consideración de Masciangioli y Zhang cuando se preguntan si las nanoestructuras serán capaces de autoensamblarse en el medio ambiente tal y como lo pueden hacer en 
el laboratorio y, en su caso, qué sería de éstas y cuál serían sus impactos ambientales y a la salud. Véase: Masciangioli y Zhang, 1 de marzo de 2003. Op cit: 107A.

65 Sarewitz, Daniel y Woodhouse, Edward. "Lo pequeño es peligroso" en, Foladori, Guillermo e Invernizzi, Noela (coord). Nanotecnologías Disruptivas. Impactos Sociales de las Nanotecnologías. Porrúa. México, 2006: 158 .

66 Food and Drug Administration. Nanotechnology. Nanotechnology Task Force. EUA, 25 de julio de 2007.

67 Martin Schmid, administrador de la división de nanotubos de carbono de Bayer Material Science AG (Alemania), señalaba al respecto de los tipos y montos de materiales empleados en la producción de nanotubos multicapa (denominados por la empresa Baytubes) que eso es información confidencial. Lo único que se puede saber es que se hacen uso de cantidades indefinidas de gases de hridrocarbono, gases inertes (¿?) y catalíticos (¿?). Comunicación personal, 25 de julio de 2006.

68 Roco, Mihail. "Progress in Gobernance of Converging Technologies Integrated from the Nanoscale." Annals of the New York Academy of Science. Vol. 1093. EUA, diciembre de 2006: 1-23.

69 Nordmann, Alfred (rapporteur). Converging Technologies - Shaping the Future of European Societies. Comisión Europea. Bruselas, 2004: 24-30.

70 Sin autor, "Nanodollars". New Scientist. EUA, 25 de febrero de 2006: 25; Sin autor, "Nano safety call". New Scientist. EUA, 11 de febrero de 2006: 7. Datos incompletos de Rejeski vertidos ante la Cámara de Representantes de EUA en 2005 hablan de un monto de unos 23 millones de dólares en más de 150 proyectos en medioambiente, salud y seguridad de las nanotecnologías en 8 agencias distintas en EUA, Canadá y la Unión Europea (Rejeski, David. "Environmental and Safety Impacts of Nanotechnology: What Research is Needed?”. Pronunciamiento ante el Comité de Ciencia de la Casa de Representantes. EUA, 17 de noviembre de 2005). 